\title{
REDUCED-ORDER $H_{\infty}$ CONTROLLERS BASED ON IMPROVED GENETIC ALGORITHM
}

\author{
Yuanwei Jing*, Wei Pan*,**, \\ and Georigi M. Dimirovski ${ }^{* * *, * * * *}$
}

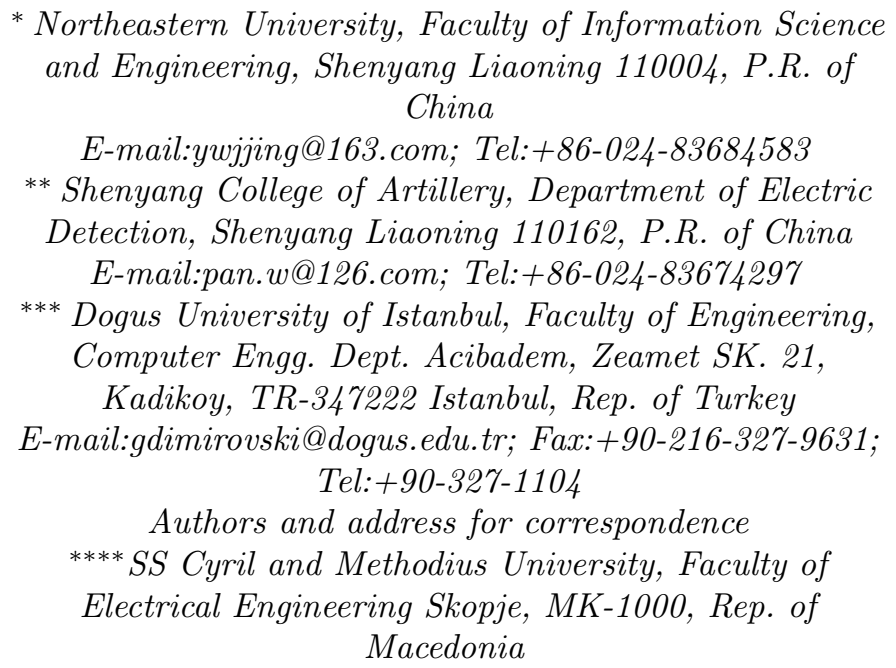

\begin{abstract}
An approach of reduced-order $H_{\infty}$ controller for a class of linear continuous dynamic systems is presented based on Genetic Algorithm. Necessary and sufficient conditions are given, in terms of linear matrix inequality, for the existence of controller. A rank condition is changed to object function of genetic algorithm(GA). The minimum order $n_{k}$ of the controller and a corresponding parameter pair $(X, Y)$ of positive definite matrix are obtained by means of searching the object function. And then the reduced-order $H_{\infty}$ controllers are constructed. In this paper, the code is float, the selection operator is rankbased fitness assignment and elitist model, the crossover operator is improved real cross, the mutation operator is real mutation. The simulation results show that the controller has the same control effect as all-order $H_{\infty}$ controllers based on MATLAB. Copyright (c) 2005 IFAC
\end{abstract}

Keywords: Improved Genetic Algorithm, Linear Matrix Inequality (LMI), Reduced-order $H_{\infty}$ controllers, All-order $H_{\infty}$ controllers, float code

\section{INTRODUCTION}

It is not easy to come true in engineering because the order of $H_{\infty}$ controller is higher or equal to the order of the object commonly. So it is significant in engineering to discuss the reduced- order $H_{\infty}$ controller. Design problems of it can be expressed as a set of linear matrix inequality adding a rank-restricted condition of matrix (Jwasaki et al., 1994), (Gahinet et al., 1994), (Pan et al., 2004), while the added rank-restricted condition is not a protruding restriction. There- 
fore, reduced-order $H_{\infty}$ controller is a non-linear and non- protruded optimization problem. At present, there are not ordinary methods to solve it except for numerical value methods, such as eliminate method and variable substitute method (Grigoriadis et al., 1995). But, many restriction conditions about object in eliminate method, and substitute formula is difficult to find in substitute method because of complex non-linear relation among $H_{\infty}$ controller variables.

GA is a kind of process searching the optimized solution algorithm that simulates nature genetic mechanism and biological evolutionism. The characteristic of GA is that it can find the optimized solution even if any information of the solved question almost unneeded while only the information of object function needed. And also it is not restricted whether the searching space is continuous or differentiable. GA is an effective optimized searching algorithm which depends on colony searching strategy and the individual information exchanging and has not relation with the grads information. It is suitable to deal with complex non-linear searching optimization question which is difficult to solve for traditional searching optimization method. So GA provides a kind of an effective numerical value method for solving the above non-linear and non-protruding optimization questions.

A kind of reduced-order $H_{\infty}$ controllers design methods based on GA is put forward aiming at linear time-invariable continuous dynamic systems. The method makes the rank of the matrix as small as possible by optimizing the matrix so that the orders of the $H_{\infty}$ controllers are reduced.

\section{PROBLEM DESCRIPTION}

Consider a singular discrete system. The statespace description is

$$
\begin{aligned}
& \dot{x}=A x+B_{1} w+B_{2} u \\
& z=C_{1} x+D_{11} w+D_{12} u \\
& y=C_{2} x+D_{21} w+D_{22} u
\end{aligned}
$$

where $X \in R^{n}, u \in R^{m}, y \in R^{p}, z \in R^{r}$ and $w \in R^{q}$ are state, control input, measure output, controlled output and outer disturbance respectively.

Design an $H_{\infty}$ output feedback controller for system (1):

$$
u(s)=K(s) y(s)
$$

then,

$$
\begin{aligned}
& \dot{\tilde{x}}=A_{k} \widetilde{x}+B_{k} y \\
& u=C_{k} \widetilde{x}+D_{k} y
\end{aligned}
$$

which makes the closed loop system stable and the closed loop function $\|T w z\|_{\infty}$ from disturbance input $\omega$ to controlled output $z$ less than the given positive number $\gamma$.

Reference (Xin et al., 1996) gives the sufficient and necessary condition that the $H_{\infty}$ controller exists, if and only if symmetry positive matrix $\mathrm{X}$ and $\mathrm{Y}$ can be found to satisfy the following inequality.

$$
\begin{gathered}
{\left[\begin{array}{cc}
N_{O} & 0 \\
0 & I
\end{array}\right]^{T} M_{O}\left[\begin{array}{cc}
N_{O} & 0 \\
0 & I
\end{array}\right]<0} \\
{\left[\begin{array}{cc}
N_{C} & 0 \\
0 & I
\end{array}\right]^{T} M_{C}\left[\begin{array}{cc}
N_{C} & 0 \\
0 & I
\end{array}\right]<0} \\
{\left[\begin{array}{cc}
X & I \\
I & Y
\end{array}\right] \geq 0}
\end{gathered}
$$

where,

$$
\begin{gathered}
{\left[\begin{array}{ccc}
A^{T} X+X A & X B_{1} & C_{1}^{T} \\
B_{1}^{T} X & -I & D_{11}^{T} \\
C_{1} & D_{11} & -I
\end{array}\right]=M_{O},} \\
{\left[\begin{array}{ccc}
A Y+Y A^{T} & Y C_{1}^{T} & B_{1} \\
C_{1} Y & -I & D_{11} \\
B_{1}^{T} C_{1} & D_{11}^{T} & -I
\end{array}\right]=M_{C}}
\end{gathered}
$$

Note $N_{O}$ and $N_{C}$ are matrixes constructed by any set of fundamental vector as column vectors in the subspace $\operatorname{ker}\left(\left[\begin{array}{ll}B_{2}^{T} & D_{12}^{T}\end{array}\right]\right)$ and $\operatorname{ker}\left(\left[\begin{array}{ll}C_{2} & D_{21}\end{array}\right]\right)$ respectively.

For the reduced-order $H_{\infty}$ controller, it is necessary to add a fourth inequality, that is

$$
\operatorname{rank}\left[\begin{array}{cc}
X & I \\
I & Y
\end{array}\right] \leq n_{k}+n
$$

where $n, n_{k}$ are the ranks of system model and controller respectively.

The added rank restricting condition is not a protruding restriction which is difficult to solve the symmetry positive matrix $X$ and $Y$. Therefore, we convert the problem to solve the minimal rank problem in satisfied LMI of formula (4)-(6).

Define object function for GA

$$
\psi(X, Y)=\sum_{i=1}^{n-n_{k}} \lambda_{i}
$$

where, $\lambda_{1} \leq \cdots \leq \lambda_{n-n_{k}}$ express the minimal eigenvalue of matrix $\left[\begin{array}{cc}X & I \\ I & Y\end{array}\right]$. Then, the design of the reduced-order $H_{\infty}$ controller is converted to looking for the minimal $\varphi(X, Y)$ satisfied LMI of formula (4)-(6). So, the existence of reduced-order $H_{\infty}$ controller is equivalent to the existence of the minimal value of $\varphi(X, Y)$ with the value 0 . 


\section{SOLUTION PROCESS OF GA}

Combining the discussed problems in this paper, the operating process of GA is as follows.

\subsection{Code}

Code(Fogel et al., 1994) converts the parameters in question space to chromosomes or individuals which formed by genes in some structure in genetic space, that is mapping from question space to GA space.

This paper adopts real number coding project, because it is high-precision, natural and intuitionistic. All items in the two optimized matrixes parameters code straightly and make up of $P$.

\subsection{Enactment of original colony}

The individual in original colony is generated at random, which is the start of GA.

Suppose that system(1) does not exist control input and measure output. Then in system model, assume that $B_{2}=0, C_{2}=0, D_{12}=0, D_{21}=0$, so, we have $N_{O}=I, N_{C}=I$.

It is easily to know, under the condition, the formula (4)-(6) can be simplified as

$$
\begin{gathered}
{\left[\begin{array}{ccc}
A^{T} X+X A & X B_{1} & C_{1}^{T} \\
B_{1}^{T} X & -I & D_{11}^{T} \\
C_{1} & D_{11} & -I
\end{array}\right]<0} \\
{\left[\begin{array}{ccc}
A Y+Y A^{T} & Y C_{1}^{T} & B_{1} \\
C_{1} Y & -I & D_{11} \\
B_{1}^{T} C_{1} & D_{11}^{T} & -I
\end{array}\right]<0} \\
{\left[\begin{array}{cc}
X & I \\
I & Y
\end{array}\right] \geq 0}
\end{gathered}
$$

where, (9) and (10) are equal, and $Y=X^{-1}$

$N$ individuals $P_{j}$ can be achieved from inequality (9) and inverse matrix can construct the original colony.

\subsection{The solution of fitness function}

The evaluation of fitness function is the basis of the choice of operation, and the design of fitness function influences the performance of GA straightly.

For the minimal rank optimized question in this paper, define fitness function as follows:

$$
f=\frac{1}{\varphi(X, Y)+e p s}
$$

where, eps is a small positive value .

\subsection{The design of GA operation}

\section{i. Selection}

Selection is to choose superior individuals and eliminate through selection or contest the inferior individuals from colony. The aim of it is to inherit the optimized individuals to the next generation. This paper adopts rank-based fitness assignment and protection of the optimized individuals of elitist model.

After calculating the fitness degree of every individual, the rank-based fitness assignment arranges individual order according to fitness degree, and then distributes probability form which is designed in advance for the individuals orderly as each selecting probability.

Elitist model does not carry out inheritance operation while copy to the next the generation straightly for the individual with the highest fitness degree, which can guarantee the optimized solution of some generation undestroyed.

\section{ii. Crossover}

Real cross includes discrete recompose, midst recompose and linear recompose etc. But the above methods have an obvious limitation, that is, when new individuals come into being, if the two bodies of father generation have the same symbols(equal in great probability), then filial generation make for increscent direction of coordinate absolute value, which go against fast convergence of optimization. So, the improved real cross(Srinivas et $a l ., 1994)$ operator is constructed.

The improved real cross operator is described as follows:

$$
\begin{aligned}
& X_{1}^{\prime}=b_{1} X_{1}+b_{2} X_{2} \\
& X_{2}^{\prime}=b_{2} X_{1}+b_{1} X_{2}
\end{aligned}
$$

where, $b_{1}=0.5+b, b_{2}=0.5-b,(b=0.95 \beta$ or $b=\beta), \beta$ is random in $[0,1]$.

iii. Mutation

The essence of mutation(Zitzler et al., 1999) is to look for individuals' diversity in colony and improve algorithm local random searching ability.

It is difficult to choose the step of mutation, the optimized step is determined under the exact condition. Mutation step will change automatically in optimizing process according to colony evolvement process.

Mutation step is given in the following formula:

$$
X^{\prime}=X \pm 0.5 L \Delta
$$


where, $X$ and $X^{\prime}$ is individual before mutation and after mutation respectively, $\Delta=\sum_{i=0}^{m} \frac{a(i)}{2^{i}}$, $a(i)$ is 1 as the probability of $\frac{1}{m}$, and gets 0 as the probability of $1-\frac{1}{m}$. In the paper, $\mathrm{m}=20, L$ express difference between the maximal value and the minimal value of individuals in generations.

The designing process of reduced-order $H_{\infty}$ controller is as follows. Searching is stated from $n_{k}=$ $n-1$, fixing $n_{k}$ and looking for parameters $(X, Y)$ of object function $\varphi(X, Y) \leq \epsilon$. If it is found, then let $n_{k}=n-1$, and repeat the previous process. Otherwise if it still can not be found after generations, it shows that there is not the optimized solution satisfied object function $\varphi(X, Y) \leq \epsilon$. Then algorithm is end. The flow chart is showed in Fig. 1.

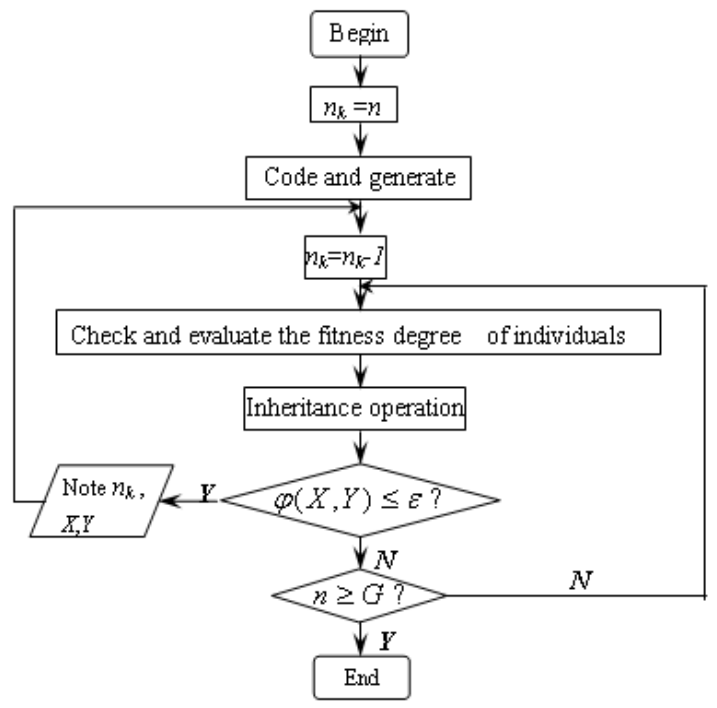

Fig. 1. Flow char of GA

The optimized solution and the order of controller are got by using the above algorithm, then the controller is designed with the method in reference (Jwasaki et al., 1994).

\section{EXAMPLE}

Consider the following linear time invariable continuous dynamic system, in which the parameters of every object is as follows.

$$
A=\left(\begin{array}{cccc}
0 & -0.2 & -0.25 & -1.0 \\
-1.0 & -2.0 & 1.0 & 0 \\
-1.0 & -0.1 & 0.85 & -1.0 \\
-0.25 & -0.5 & 0 & -0.25
\end{array}\right)
$$

$$
\begin{aligned}
& B_{1}=\left(\begin{array}{cccc}
0.25 & 0 & 0 & 0 \\
0 & 0.25 & 0 & 0 \\
0 & 0 & 0.25 & 0 \\
0 & 0 & 0 & 0.25
\end{array}\right) \\
& B_{2}=\left(\begin{array}{cc}
1.0 & 0 \\
1.0 & 0 \\
0 & 1.0 \\
0 & 1.0
\end{array}\right) \\
& C_{1}=\left(\begin{array}{llll}
1 & 0 & 0 & 0 \\
0 & 0 & 1 & 0 \\
0 & 0 & 0 & 1 \\
0 & 1 & 0 & 0
\end{array}\right), C_{2}=\left(\begin{array}{cccc}
20 & 0 & 0 & 0 \\
1.2 & 1.8 & 0 & 0 \\
0 & 0.25 & 27 & 0 \\
0 & 0 & 0 & 10
\end{array}\right) \text {, } \\
& D_{11}=\left(\begin{array}{cccc}
0.1 & 0 & 0 & 0 \\
0 & 0.1 & 0 & 0 \\
0 & 0 & 0.1 & 0 \\
0 & 0 & 0 & 0.1
\end{array}\right), D_{12}=\left(\begin{array}{ll}
0 & 0 \\
0 & 0 \\
1 & 0 \\
0 & 1
\end{array}\right) \\
& D_{21}=\left(\begin{array}{cccc}
1 & 0 & 0 & 0 \\
0.1 & 0.1 & 0 & 0 \\
0 & 0 & 0 & 0 \\
0 & 0 & 0 & 0
\end{array}\right), D_{22}=\left(\begin{array}{ll}
0 & 0 \\
0 & 0 \\
0 & 0 \\
0 & 0
\end{array}\right) \text {. }
\end{aligned}
$$

The system exists a pair of unstable poles $0.9857 \pm 0.4587 i$ distributing at right-half virtual axis of plural plane.

$H_{\infty}$ controller is get making use of hinflmi() function in LMI control toolbox of MATLAB, the order of controller and model are 4. Performance of system optimization full-order $H_{\infty}$ controller is $\gamma_{\text {opt }}=0.6019$, step response curve of closed loop is showed in Fig. 2.
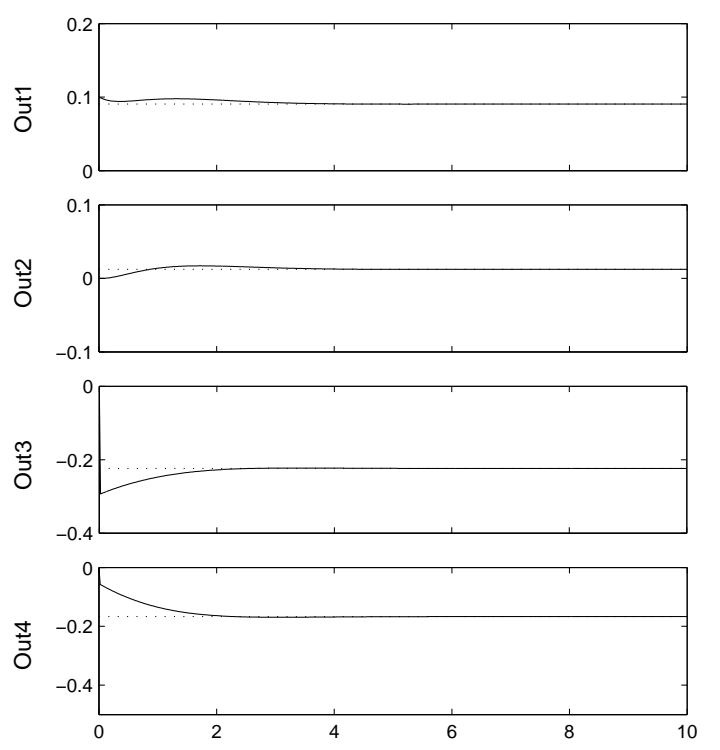

Fig. 2. Step response of all-order $H_{\infty}$ controllers

Reduced-order $H_{\infty}$ controller is solved by making use of the algorithm proposed in the paper. Parameters are designed as follows: $N=12--$ colony model, $G=100--$ the maximal value of evaluation generation, $P_{c}=0.65--$ cross probability, $P_{m}=0.001--$ mutation probability, $\varepsilon=$ $10^{-10}--$ ending condition. 
In the process of searching the minimal order of controller, when evolution has happened 19 times, that is $n_{k}=3$, object functions of the optimized individuals can be achieved $2.8 \times 10^{-11}$, while evolution has happened 100 times, that is $n_{k}=2$, object functions of the optimized individual only get 1.8842. So, the minimal order of controller is 3 in the example, and 3 -order $H_{\infty}$ controller is designed according to corresponding value of $(X, Y)$. The optimized $H_{\infty}$ controller performance of the system is $\gamma_{o p t}=0.7854$, then step responding curve of the closed loop system is shown as Fig. 3 .
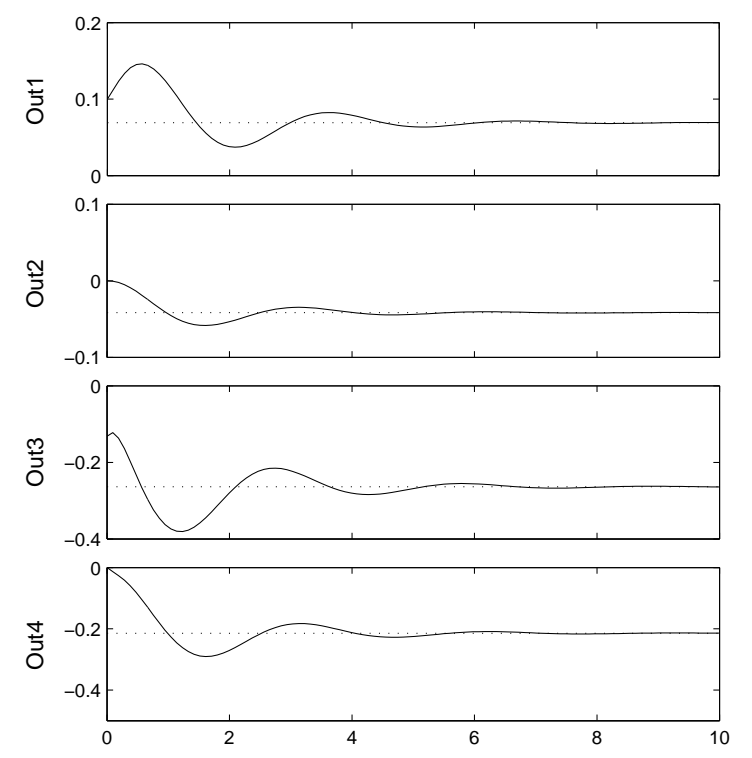

Fig. 3. Step response of all-order $H_{\infty}$ controllers

Surge frequency of designed reduced-order $H_{\infty}$ controller is high and stable time is long, but order of controller is reduced which makes engineering realization possible. With comparison in Fig. 2 and Fig. 3, reduced-order $H_{\infty}$ controller and fullorder $H_{\infty}$ controller designed with MATLAB get the same control purpose.

\section{CONCLUSIONS}

The design method of reduced-order $H_{\infty}$ controller based on GA is put forward in the paper. The minimal rank of a set of matrixes with LMI restriction is solved by GA, and then the minimal order $n_{k}$ of reduced-order $H_{\infty}$ controller and the corresponding parameter $(X, Y)$ are obtained, thereby the reduced-order $H_{\infty}$ controller is designed.

GA is an effective optimized searching algorithm, which regards object function value as searching information while it does not depend on grads information, it deals with coding set of decisionmaking variable straightly not rather than the real value of its own, which guarantees the applicability not only for control problems of singular object but also for non-singular.

The results in example show that the designed controller not only reduces the order but also stabilizes the system preferably.

\section{Acknowledgements}

Authorities of Northeastern University of Shenyang and SS Cyril and Methodius of Skopje are acknowledged for long supporting the academic collaboration between Professors Jing and Dimirovski. Also, Dimirovski is grateful to Dogus University for continuing support of his international activities since he joined this university.

\section{REFERENCES}

Fogel, D.B. (1994). An introduction to simulated evolutionary optimization. IEEE Trans on Neural Networks. 5(1),3-14.

Gahinet, P. and Apkarian, P. (1994). A linear inequality approach to $H_{\infty}$ control. Int $J$ Robust and Nonlinear Control. 4(1),421-448.

Grigoriadis, K.S. and Skelton,R.E (1995). Low order control design for LMI problems using alternationg projection methods. Automatica. 31(8), 1117-1125.

Jwasaki, T. and Skelton R.E. (1994). All controller for the general $H_{\infty}$ control problem: LMI existence conditions and state space formulas. Automatica. 30(8),1307-1371.

Pan, W. and Jing, Y.W. (2004). Reduced-order $H_{\infty}$ controllers based on Genetic Algorithm. Journal of Northeastern University(Natural Science). 25(6),519-522.

Srinivas, M. and Patnail L.M. (1994). An adaptive probabilities of crossover and mutations in Gas. IEEE Trans on SMC. 24(4),656-667.

Xin, X., Guo, L. and Feng, C.B. (1996). Reducedorder controllers for continuous and discrete time singular $H_{\infty}$ control problems based on LMI. Automatica. 32(10),1601-1609.

Zitzler,E. and Thiele, L. (1999). Multi-Objective Evolutionary Algorithms: A Comparative Case Study And the Strength Pareto Approach. IEEE Transactions of Evolutionary Computation. 3(4),257-271. 